Evolution of Intermediate and Low Mass Binary Systems

P. P. Eggleton

October 27, 2005

A Life with Stars

Amsterdam, Netherlands

August 22, 2005 through August 26, 2005 
This document was prepared as an account of work sponsored by an agency of the United States Government. Neither the United States Government nor the University of California nor any of their employees, makes any warranty, express or implied, or assumes any legal liability or responsibility for the accuracy, completeness, or usefulness of any information, apparatus, product, or process disclosed, or represents that its use would not infringe privately owned rights. Reference herein to any specific commercial product, process, or service by trade name, trademark, manufacturer, or otherwise, does not necessarily constitute or imply its endorsement, recommendation, or favoring by the United States Government or the University of California. The views and opinions of authors expressed herein do not necessarily state or reflect those of the United States Government or the University of California, and shall not be used for advertising or product endorsement purposes. 


\title{
Evolution of Intermediate and Low Mass Binary Systems
}

\author{
Peter P. Eggleton ${ }^{\text {a }}$

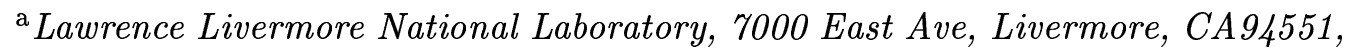 \\ $U S A$
}

\begin{abstract}
There are a number of binaries, fairly wide and with one or even two evolved giant components, that do not agree very well with conventional stellar evolution: the secondaries are substantially larger ('oversized') than they should be because their masses are quite low compared with the primaries. I discuss the possibility that these binaries are former triples, in which a merger has occurred fairly recently in a short-period binary sub-component.

Some mergers are expected, and may follow a phase of contact evolution. I suggest that in contact there is substantial transfer of luminosity between the components due to differential rotation, of the character observed by helioseismology in the Sun's surface convection zone.
\end{abstract}

Key words: Binary stars; stellar evolution; triple stars

\section{Introduction}

The definition of 'intermediate' and 'low' masses for stars is not at all clear, but I will take 'intermediate' to mean masses such that a star ends up as a white dwarf rather than as a neutron star; and further that 'low' begins when stars develop cool, deep red-giant envelopes shortly after leaving the main sequence. Roughly, this suggests ranges of $\sim 2-8 M_{\odot}$ and $\lesssim 2 M_{\odot}$, respectively. For the most part I will also exclude 'very low' masses, $\lesssim 1 M_{\odot}$, since at these masses stars are barely capable of nuclear evolution.

The significance of deep convective envelopes is that these can apparently be sites of dynamo activity, and this can lead to stellar winds and magnetic braking. Although single stars can be expected to be rather slow rotators once they become red giants, tidal friction in binaries can produce substantially more rapid rotation, and rotation appears to correlate strongly with magnetic activity. Thus mass loss and consequential angular momentum loss, the latter affecting the orbit and not just the rotation, because of tidal friction, 
can be expected to be significant in the evolution of low-mass stars.

The fact that binaries containing cool stars can be active, and so potentially subject to magnetic braking and orbital shrinkage, means that even 'very low mass' binaries are in fact objects of interest. Two K/M dwarfs may spiral in slowly, and merge - perhaps with an intermediate stage as a contact binary - to form a single star which may well be capable of nuclear evolution. In fact, since $\mathrm{K} / \mathrm{M}$ dwarfs are much more common than any other kind of star, it is likely that most binary interaction is of this form. But it would take a long article to do justice to this topic as well.

\section{Some Evolutionary Pro- cesses}

In addition to obvious things such as nuclear physics, the equation of state, etc., stellar evolution computations need to include some approximation to at least the following items:

(1)* Rotation $\Omega$, say, implying a Rossby no. which is a function of $\Omega, M, R$ and $L$

$(2)^{*}$ Differential rotation, as observed in the Solar convective envelope

(3)* Magnetic activity and cycles, driven by a combination of rotation, differential rotation, and turbulent convection in the atmosphere

(4)* Stellar wind driven by dynamo activity - and in addition several types not specifically 'dynamodriven'

(5)* Magnetic braking

(6) Selective diffusion

(7) Mixing driven by RayleighTaylor instability

(8)* Tidal effects, tidal friction

$(9)^{*}$ Eccentricity, synchronisation, circularisation

(10)* Roche-lobe overflow (RLOF), spin-up of gainer

(11)* Partial wind accretion; bi-polar re-emission

$(12)^{*}$ Shallow common envelope: contact binaries with heat transfer in envelope, which I believe is due to differential rotation

(13) Mergers, of several types

(14) Deep common envelope: mergers, near-mergers, nowherenear-mergers

(15) Rapid envelope ejection

(16) Supernova explosions with or without kicks

(17) Dynamical interaction in clusters

(18) Modification of inner orbits and spins, in triples

(19) Mergers in triples, leading to unusual binaries.

An asterisk indicates that my code ' $E V$ ' currently includes these, sometimes at a rather elementary level of approximation. The processes listed above are crudely divided into three groups: those that operate in single stars, those that operate in binaries, and those that require higher multiplicity. My current EV allows the possibility, in binaries, of solving both component stars simultaneously. By this I mean that if $N$ 
equations describe each component, then $2 N$ simultaneous equations are solved by a standard linearisation and Newton-Raphson iterative procedure.

Many of the processes listed are 'nonconservative', i.e. they can remove either mass or angular momentum, or both, from the system. There is a long history of modeling conservative evolution, but much less of modeling non-conservative effects. Partly this is because it is necessary to include the effects in both components. I believe this will be made easier with my new code which solves both components simultaneously.

\section{Methodology}

How should we proceed to test such 'non-conservative' models? Two alternatives are:

(a) do a large grid of models (as by Nelson \& Eggleton 2001 - NE01 - for conservative evolution), or

(b) do a case-by-case comparison between observed systems and models.

NE01 concluded that conservative evolution could account quite well for several well-observed Case A 'hot Algol' systems. But systems with cool components $(\mathrm{F} / \mathrm{G} / \mathrm{K})$ were not well accounted for, and they probably demand some non-conservative processes. Also very hot and/or luminous systems (Of stars, WRs, LBVs, ...) are not likely to be well acounted for, but are outside the scope of this talk.

A large grid of non-conservative models would only be justifiable if we were fairly confident of the strengths of the various effects. There are at least half-a-dozen, and quite probably more, coefficients and exponents involved that are little more than guesses. Our grid should presumably include at least two values of each uncertain parameter, and thus would be immense. Therefore I suggest that (b) is the way to proceed for the moment.

Before embarking on this however, I have to report that $\mathrm{u}$ Her, an intermediate-mass semidetached binary which was found (NE01) to fit tolerably well to the conservative model, is now more of a problem since the masses of the two components have been revised upwards by about $50 \%$ (Hilditch 2005). It is a fairly short-period 'hot Algol', that must be an example of Case A RLOF. We (NE01) found that Case A is actually quite a complicated collection of quite radically different scenarios. We identified eight major sub-cases, that can occur in different regions of the three-dimensional parameter space of total mass, mass ratio and period. Some of these sub-cases lead to contact binaries, where the gainer reaches its own Roche lobe on either a dynamical timescale (Case AD), a thermal (rapid) timescale (Case AR) or a nuclear (slow) timescale (Case $\mathrm{AS})$. In these sub-cases it is not possible for the mass ratio to become very extreme before contact is reached; this usually happens before the mass ratio drops to $\sim 0.4$ (if by definition 
the mass ratio was greater than unity to start with). Others manage to avoid contact (Case AN), and evolve to the more extreme mass ratios $(0.3$ - 0.1) typical of normal Algols. But the region of parameter space where Case AN happens is quite restricted, and does not include those systems with the shortest periods at age zero.

The short period of u Her made it a rather marginal example of Case $\mathrm{AN}$, in the analysis of NE01. The observed mass ratio of $\sim 0.33$ is too low for Case AS, which would normally reach contact before such a ratio is reached. The period was marginally consistent with the AN/AS boundary, and in Case AN such a mass ratio was accessible. But with larger masses (and consequential larger radii), the period at the AN/AS interface is shifted upwards, and so it is much more difficult to argue that $\mathrm{u}$ Her is a marginal Case AN system, even though the mass ratio demands it.

It may be possible to tweak the theoretical models back into agreement, for example by supposing that there is less convective overshooting than we assumed (Pols et al. 1997, Schröder et al. 1997) on the basis of modeling non-interactive binaries. However, I feel that an area which I had hoped was reasonably well understood is now less well understood, at least by me.

I suggested above that for the present, and in the context of lowmass systems, it is best to try a case-by-case comparison, to see if any general principles emerge, e.g. that mass-loss rates, or tidal friction, must usually be greater/smaller than the 'canonical' model suggests. Let us consider the relatively straightforward case of a well-evolved binary which has nevertheless not reached RLOF: HR 2030 (Griffin \& Griffin 2000). This has parameters: K0IIb + $\mathrm{B} 8 \mathrm{IV}, 4+4 M_{\odot}, 41+5.9 R_{\odot}, 66.5 \mathrm{~d}$, $e=0.018 \pm 0.006$. The mass ratio $q$ is $1.00 \pm 0.03$. The period is so short that the primary must still be in the Hertzsprung gap, approaching He ignition - although RLOF should happen even sooner. The primary is quite active: has it lost significant mass by rotationally-enhanced stellar wind? It cannot have lost much. The secondary is evolved almost to the end of the MS, and this can happen only if the original mass ratio was close to unity (but see OW Gem, later!).

My canonical non-conservative model works reasonably well. I can start with $4.03+3.96 M_{\odot}, 80.7 \mathrm{~d}$, $e=0.3$, and by the time both components have reached their observed radii (at $171.4 \mathrm{Myr}$ ) the masses are $3.985+3.96 M_{\odot}$. The eccentricity is however down to zero, having reached 0.02 when the primary was only $27 R_{\odot}$. Griffin \& Griffin (2000) consider at length whether the eccentricity is real, or a possible artefact of distorted surfaces (the Barr effect). Their conclusion is a firm 'maybe'.

I think the comparison shows that:

(1) The tidal-friction model cannot be a strong overestimate: if it were, the orbit would be conspicuously eccentric. 
(2) The rotationally-dynamo-driven wind model cannot be a strong underestimate. Ten times as much mass loss can certainly be ruled out.

Another system where the primary has evolved well into the Hertzsprung gap, but not beyond, is o Leo (Hummel et al. 2001, Griffin 2002): F9III $+\mathrm{A} 7 \mathrm{~m}, 2.12+1.87 M_{\odot}, 5.7+2.6 R_{\odot}$ $14.5 \mathrm{~d}, e=0$. My canonical model does not achieve the observed circularisation, but would if tidal friction were strengthened by a factor of 3 or so. This is not necessarily inconsistent with HR 2030. The present radii are fairly consistent with the masses, at age $933 \mathrm{Myr}$.

A third system, RZ Eri, shows rather more clearly the effect of some non-conservative processes. Its parameters are: K2III + F5m, 1.62 $+1.68 M_{\odot}, 7.0+2.8 R_{\odot}, 39.3 \mathrm{~d}$, $e=0.35$ (Popper 1988). The redgiant primary is an active, spotted (RSCVn-like) star, and has a rotational period of $31.4 \mathrm{~d}$. Note that the larger and presumably more evolved star is slightly the less massive; but this is comfortably consistent with my non-conservative model, because the giant rotates rapidly and so is quite active and windy.

However there is at least a small problem with this system. We would expect that tidal friction has established pseudo-synchronism (Hut 1981) in this not-yet-circularised system. But the pseudo-synchronous rotation period (23d) is substantially smaller than what is observed. This might have two explanations: (i) tidal friction is rather weaker than expected, or (ii) magnetic braking is rather stronger than expected, and is slowing down the rotation despite the fact that tidal friction is trying to speed it up.

One can continue with a number of other systems. But possible progress runs up against a rather alarming fact: there are a few systems with such perverse data that no amount of tinkering with non-conservative fudge-factors is likely to resolve the difficulties. Let me describe two systems with data which are of much the same quality as the three above.

The system $\gamma$ Per is considerably wider than these three, and so any kind of binary interaction in the past (apart from the basic gravitational one!) seems out of the question. Its parameters are: G8III + A3V, $2.5+1.86 M_{\odot}, 21+4 R_{\odot}, 5350 \mathrm{~d}$, $e=.79$ (Pourbaix 1999). Clearly in $\gamma$ Per the secondary is quite substantially evolved. It is about 2.6 times the radius expected for an unevolved star of that mass. Yet with $q=1.34$ we should expect hardly any evolution in the secondary. It is clearly not a question that stellar wind has been important, because that would act in the opposite direction.

Far more extreme is OW Gem (Griffin \& Duquennoy 1993, Terrell et al. 2003). Its parameters are: F2Ib-II + G8IIb, $5.8+3.9 M_{\odot}, 30+32 R_{\odot}$, $1259 \mathrm{~d}, e=.52$ It is not so wide as $\gamma$ Per, but still much too wide (and eccentric) for the probability of any serious interaction so far. The 'secondary', if that term has any meaning in this context, is larger than the 
'primary', and yet should be almost completely unevolved according to the system's mass ratio, which is even larger than in $\gamma$ Per.

R. E. M. Griffin (p.c. 1998) has referred to the problem as 'oversized secondaries'. OW Gem is a very extreme case.

I have drawn up a list of about 20 systems with (a) one component (at least) being a giant, (b) an orbit sufficiently wide that RLOF (currently or formerly) is unlikely, and (c) data of reasonably high quality. Two of them have $q$ less than 1 ( $\mathrm{Z}$ Her, RZ Eri). This can be explained by 'binary-enhanced stellar wind' from the more evolved component, reasonably in accord with my canonical model. Six more of them ( $\delta$ Sge, $\zeta$ Aur, QS Vul and V1488 Cyg in addition to $\gamma$ Per and OW Gem) have oversized secondaries: the first four are not as extreme as $\gamma$ Per, and yet they are apparently outside the limits that stellar evolution suggests. The remaining 12 are not a problem, or at least not by much. In them, the ratio $R_{2} / R_{\mathrm{ZAMS}}\left(M_{2}\right)$ correlates with $q \equiv M_{1} / M_{2}$ pretty much as expected.

Some years ago (Eggleton 1996) I suggested that OW Gem is the result of a merger of a close binary which is (or was) one component of the current wide binary. The triple started with parameters $\quad\left(\left(3.9+1.9 M_{\odot}\right.\right.$; $\left.\sim 3 \mathrm{~d})+3.9 M_{\odot} ; 1259 \mathrm{~d}\right)$ and then, when both the massive components evolved into the Hertzsprung gap at more-or-less the same time (cf. HR 2030), the short-period pair ran into severe RLOF and merged. I estimate that $\sim 1-2 \%$ of stellar systems are triples in which the outer period is moderately short, $\lesssim 30$ yr. About $20 \%$ of stellar systems (single stars being systems, as well as binaries and triples) are binaries in the same period range. It therefore does not look promising that 6 out of 20 binaries are former triples.

However, this does not allow for the probability (I suggest) that former triples with a merged component should be unusually conspicuous as binaries. If $\gamma$ Per were 'normal', so far as its red-giant component is concerned, its A-type component would have less than half of the radius, and only $\sim 20 \%$ of the luminosity, that it now has. It is quite possible that $\gamma$ Per would not have been recognised as a binary, or at least only as a single-lined binary. There may therefore be a selection effect which favours the discovery of widish binaries that are former triples.

I suggest that a merger is a likely result of RLOF if (a) the period is short (a few days), and (b) the mass ratio is moderately large, $q \gtrsim 2$ and perhaps even $\gtrsim 1.5$. In such circumstances RLOF is very likely to lead to a contact configuration. I believe that we still cannot be sure how contact binaries evolve (though see later), but a very plausible hypothesis is that $q$, after decreasing a little during the initial very rapid RLOF, subsequently increases and in effect $\rightarrow \infty$. Alternatively, $q$ might manage to decrease through unity and continue to zero. 
For $\gamma$ Per, I hypothesise that the system started with parameters $\left(\left(1.9+0.6 M_{\odot} ; \sim 2 \mathrm{~d}\right)+1.86 M_{\odot}\right.$; $5350 \mathrm{~d})$ Looking through lists of known triples, I cannot see one with all the properties I want, but $\beta$ Cap and V819 Her (HR 6469) have some. $\beta$ Cap can be described by ((B8V + ?; $8.68 \mathrm{~d})+$ K0II-III; $3.76 \mathrm{yr}$, Evans \& Fekel 1979), and V819 Her by $((\mathrm{F} 2 \mathrm{~V}+\mathrm{F} 8 \mathrm{~V} ; 2.23 \mathrm{~d})+$ G8IIIIV; 5.54 yr; Scarfe et al. 1994). The masses in $\beta$ Cap are estimated as $((3.3+0.9)+3.7) M_{\odot}$. If the two largest masses were interchanged, we could expect (i) a merger, and (ii) an oversized secondary - though the result would be more like $\delta$ Sge $\left(3.4+2.7 M_{\odot}\right)$ than $\gamma$ Per.

The masses in V819 Her are estimated as $((1.53+1.11)+1.84) M_{\odot}$. These are nearer the right values in total, but the mass ratio in the subsystem should be more extreme. I expect that the short-period pair in V819 Her will evolve into a respectable Algol, and the system will remain triple - rather like Algol itself.

There are of course several triples where there is ongoing RLOF that has clearly not produced a merger. Presumably these are the ones which started with $q \sim 1-1.5$ in the inner pair, and the mergers only occur if $q$ is substantially larger to start with. About 20 Algols are known among the $\sim 9000$ stars, brighter than $V \sim 6.5$, in the Bright Star Catalogue (BSC; Hoffleit \& Jaschek 1983). At least four are known to be in triples ( $\lambda$ Tau, $\beta$ Per, $\delta$ Lib and V505 Sgr) with outer periods ranging from $33 \mathrm{~d}$ to 39 yr. For every non-merger, capable of producing an Algol, we might anticipate two mergers, from systems with higher initial mass ratios. So it is not unreasonable to expect in the BSC eight systems with 'oversized secondaries'. Of the six such systems referred to above, five are in the BSC. Obviously such a calculation needs refinement, but the numbers do not seem to be grotesquely out-of-line.

We would certainly expect that the product of a merger should be in rapid rotation. Unfortunately none of the putative merger products in the 'oversized secondary' population is known to be rotating unusually rapidly. This is a problem, but in the absence (as I see it) of a better explanation for oversized secondaries I am forced to argue that the spindown of a rapidly rotating red giant that is effectively single (i.e. so far from its binary companion that tidal friction is negligible) must be rather rapid, presumably because of the enhanced activity as in RS CVn systems. There is a small population of rapidly rotating, active, yet effectively single red giants, the FK Com stars (Bopp \& Stencel 1981). They have been suggested as merger products by Webbink $(1976,1980)$. The fact that there are not many is at least consistent with the hypothesis that spin-down is fairly rapid.

If mergers among short-period, high$q$ systems are fairly common, they may have other consequences. For example, they may produce single Be stars. As is well known, they may also produce blue stragglers in clusters. It is not unreasonable to sug- 
gest that the spin-down of Be merger products is relatively slower than the spin-down of $\mathrm{G} / \mathrm{K}$ merger products, since the latters' deep convective envelopes may be more conducive to magnetic braking. Whether a merger product is a Be star or an FK Com star presumably depends on the initial period: a short period should lead to Case A and a Be star, while a longer period could lead to Case B and an FK Com star. But in addition the slow mergers of contact binaries, whether originally Case A or Case B, may produce FK Coms rather than Bes.

There is not space here to discuss the important topic of Be stars in any depth. There are certainly some Be stars which are either currentlyinteracting RLOF binaries (e.g. CX Dra $=$ HR7084) or formerlyinteracting post-RLOF binaries (e.g. $\phi$ Per and $\gamma$ Cas), and where the rapid rotation is well explained by the current or former RLOF. However I believe there are too many Be stars, particularly at early B, for this to be the universal explanation. Mergers of some Case A, high- $q$ systems may explain some more, and perhaps the rest are simply stars 'born' with unusually rapid rotation.

\section{Contact Binaries}

I indicated in Section 2 that my evolution code includes - under (12) - a model of contact binary evolution, involving heat transfer as well as mass transfer in a shallow hydrostatic common envelope. To imple- ment this effectively, I generalised the code to involve the simultaneous solution of both components. Robertson \& Eggleton (1977) had already done this, but at a time when computers were so primitive that a number of corners had to be cut (e.g. no nuclear evolution).

The evolution of contact binaries has remained a surprisingly underexplored area. Probably part of the reason for this is that numerical modeling is rather difficult, and expensive of computer time even today. Another part is that it has remained remarkably unclear what the basic mechanism is that transfers heat from one component to the other in a contact binary, and thus keeps the surface temperatures closely equal despite the very different masses. However I believe I can now supply the answer to the second point; and the first point is largely answered by the progress in computer power.

Yakut \& Eggleton (2005; YE05) recently reviewed evidence regarding the evolution of close low-mass binaries, and so I will only briefly mention a few points here. Such evolution is potentially very complex, because the timescales of magnetic braking, stellar-wind mass loss and nuclear evolution are all likely to be competitive at masses of $\sim 1 M_{\odot}$ and periods of $\lesssim 1 \mathrm{~d}$. Given that at least the first two of these three processes are very uncertain, there are many possibilities that have to be considered, although Mother Nature no doubt selects only some of these possibilities. 
I believe that heat transfer in the shallow common envelopes of contact binaries is a consequence of differential rotation, as observed fairly directly in the Sun's convective envelope (Thomson et al. 2003). An equatorial belt, of width $\pm 30^{\circ}$ and depth $\sim 30 \%$ of the Sun's radius, is seen to be rotating about $10 \%$ faster than the mean rotation of the entire Sun. A colossal flux of energy is being advected (not convected) 'sideways' by this process; a crude estimate is $\sim 3000 L_{\odot}$. Of course, this makes no difference to the Sun, since it is anyway almost completely axisymmetric. If however the Sun was in contact with a somewhat similar companion, say half its mass, sharing an envelope whose depth is about $5 \%$ of the radius - as is fairly typical for contact binaries - and if, as we would expect, the companion is also generating differential rotation in the same direction, the pair will be surrounded by an equatorial belt which can easily transfer sideways a significant amount of luminosity from the hotter star to the cooler star. For the particular pair of stars suggested, the amount of luminosity that has to be transferred is $\sim 0.3 L_{\odot}$.

I model the heat transfer with an effective energy generation rate in the contact envelope of $\pm \Delta \Omega\left(H_{1}-H_{2}\right)$, where $\Delta \Omega$ is the rate of differential rotation and $H_{1}, H_{2}$ are the enthalpies in the two atmospheres at the same level of Roche potential. The sign depends on which component is considered. Although the crude model of dynamo activity already incorporated in the code includes an estimate of, in effect,
$\Delta \Omega$, in my preliminary exploration I simply take $\Delta \Omega \sim 10^{-8} / \mathrm{s} ;$ much the same value as observed in the Sun but relatively speaking a great deal less, since the binary is rotating about 100 times faster.

The mass transfer rate in contact is modeled as depending primarily on the difference between the Roche potentials at the surfaces of the two components. However it is actually controlled by negative feedback through the fact that mass transfer influences the luminosity of each component, which in turn influences the radii. It is also in effect negative feedback which keeps the luminosity transfer in practice close to the value that is required to keep the two surface temperatures closely equal when in contact.

Fig. 1 illustrates the evolution of a binary with initial parameters $1.25+1.0 M_{\odot}, 0.47$ d. Fig. 1a shows the mass-gain rate of star 1 plotted on a quasi-logaritmic scale: values +4 and -4 correspond to values of $10^{4}$ and $-10^{4}\left(\right.$ not $\left.10^{-4}\right)$, but in the range between \pm 1 the scale is roughly linear. In fact, what is plotted vertically is $\sinh ^{-1}(\dot{M} /$ const. $)$; the value of the constant is unimportant so far as seeing the oscillatory character of the evolution is concerned. The horizontal axis is timestep number rather than time, because all the major swings seen (once the stars come into contact) occupy only $10 \%$ of the stretch of evolution shown.

Up to timestep $\sim 400$ the system is detached, so that the mass-gain rate of star 1 is negative, due to stellar 
(a) Mass Gain Rate (quasi-logarithmic)

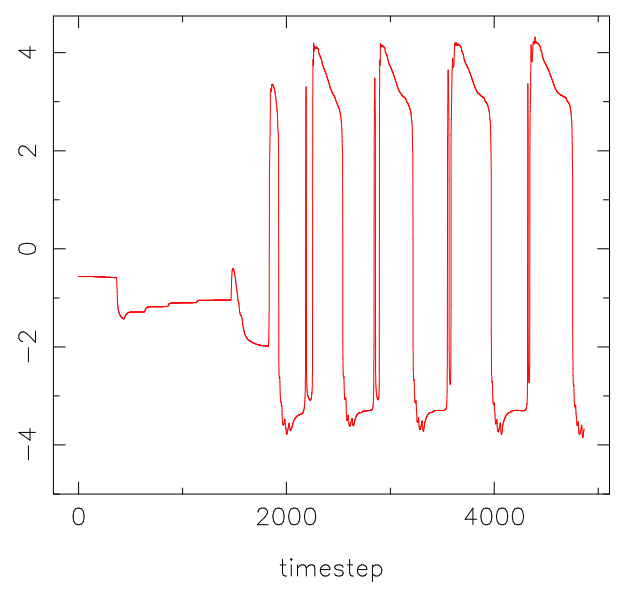

(b) $\mathrm{L}_{1}, \mathrm{~L}_{2}, 10 \Delta \log \mathrm{T}, \Delta \mathrm{L}$

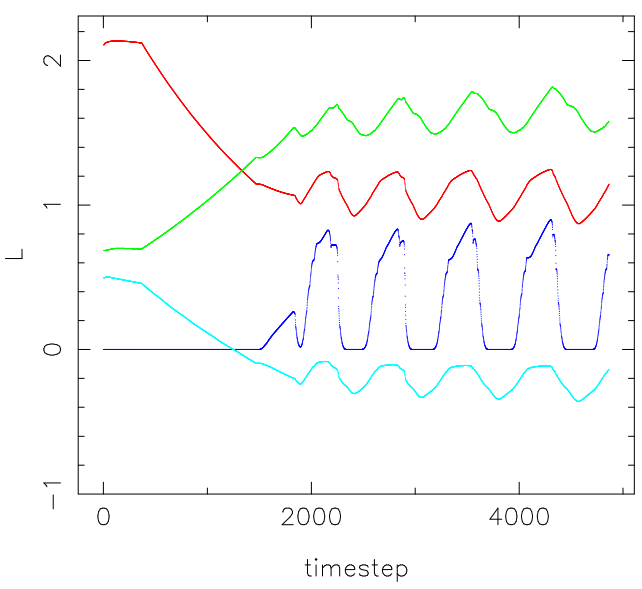

Fig. 1. The evolution of a binary with initial parameters $1.25+1.0 M_{\odot}, 0.467 \mathrm{~d}$. (a) The mass-gain rate of star 1, including wind as well as RLOF; (b) the luminosities of star 1 and star 2 , the difference in effective temperature $\left(10 \log \left(T_{1} / T_{2}\right)\right.$, and the luminosity transfer. The four curves are in order from top to bottom at the left of (b). The abscissa is timestep number rather than time, because the major swings take place in only the last $10 \%$ of the time. The mass-gain rate is on a quasi-logarithmic scale (actually, arcsinh) such that the sign is preserved, but the ordinate is roughly logarithmic for large $|\dot{M}|$. The three luminosities in (b) are in Solar units.

wind only, and rather small. Then the system becomes semidetached and the mass loss rate increases by about a factor of 10 , to roughly the nuclear timescale. Although we might expect thermal timescale RLOF here according to standard wisdom, a star of $\sim 1-1.5 M_{\odot}$ has a radius which is particularly sensitive to mass, and this allows the RLOF to proceed, unusually, on only the nuclear timescale.

However both components are so near their Roche lobes at these short periods that star 2 cannot avoid filling its own Roche lobe, and the system reaches contact at timestep $\sim 1500$. By this time the mass ratio has reversed, by a very narrow margin: the parameters are $\left(1.03+1.10 M_{\odot}, 0.38 \mathrm{~d}\right)$. Subsequent evolution shows major swings in direction of mass transfer as well as rate. These are the thermal relaxation oscillations (TROs) of Lucy (1976), Flannery (1976) and Robertson \& Eggleton (1977). Note however that in those early analyses the luminosity transfer was simply assumed to transport the right amount of heat, without a clear mechanism for this being modeled, whereas here I am modeling a specific mechanism (though only crudely) that can easily carry the modest amount of luminosity required.

Fig. 1b shows the luminosity of each component as a function of timestep number (top two curves). The luminosities pass through equality at about the same time that the masses do. The bottom curve (starting at 
the left) is the amount of luminosity transfer, which of course drops to zero in the semidetached portion of each oscillation. Note that the luminosity transfer is already included in the the luminosities plotted as the top two curves. The remaining curve in Fig. $1 b$ is the log of the ratio of effective temperatures, multiplied by 10 for legibility. The temperatures are nearly, but not quite, equal during the contact portion of each oscillation, but differ by up to $\sim 10 \%$ during the semidetached portion.

Among binaries with periods $\leq 0.5 \mathrm{~d}$, the great majority (by about 12 to 1 , YE05) have closely equal temperatures and are in contact, while a small minority have moderately unequal temperatures and are arguably semidetached. Fig. 1b would suggest that the proportion should be about 1 to 1 . However the system in Fig. 1 had unusually closely equal masses during its TRO phase, and it is possible, though not yet established, that the cycles are much more asymmetric when the mass ratios are 2 to 1 or more. the median mass ratio in contact systems is something like 4 to 1 .

It is not just by chance that the computed system had nearly equal masses. Unfortunately experience shows that it is much more difficult to compute the evolution, cyclic or not, of systems with mass ratios of even 2 to 1 , and the system of Fig. 1 is the only one I have been able to follow, so far for a few dozen cycles. The mass ratio does appear to diverge, but not by very much $(\sim 2 \%)$ in what is still a rather short time. With the eye of faith I can even claim to see a slight progression towards relatively more time in contact, as I would like.

I suspect that there must be at least three strongly different modes of evolution for low-mass (and intermediate) close binaries:

(1) Evolution fairly directly into a merger, probably requiring an initial mass ratio of $\sim 2$ or more. The rapidly-rotating, yet not very young, $\mathrm{K}$ dwarf $\mathrm{AB}$ Dor may be the remnant of such a merger (Eggleton \& KisselevaEggleton 2005); so might some Be stars.

(2) Evolution into contact before (or very shortly after, as in Fig. 1) the mass ratio can reverse itself, and then evolution, presumably with a large number of TROs, while the mass ratio increases ultimately to infinity (i.e. a merger, but a fairly slow one). This might produce both Be stars and FK Com stars, in the long term.

(3) Evolution that misses contact perhaps by a very narrow margin, and leads to Algols and post-Algols with small mass ratios; the periods may be quite short because of magnetic braking.

But, given the wealth of competing timescales (nuclear evolution, magnetic braking and mass loss, for instance), it will not be easy to tie specific observed systems to specific initial conditions. 


\section{Conclusions}

There are many binaries of low to intermediate mass that are going to need mass loss by stellar wind, and consequential angular momentum loss, as part of their evolutionary history. Unfortunately even the inclusion of these processes will not explain some systems, such as those with 'oversized secondaries'. We may have to include both triple stars, and the possibility of mergers, in order to populate the solar neighbourhood with the kind of systems that are actually seen.

For those systems which evolve into contact binaries, there is a good prospect that the heat transfer between the components can be accounted for relatively simply, as the consequence of Solar-like 'equatorial acceleration', i.e. differential rotation.

\section{Acknowledgments}

This study has been supported under the auspices of the U.S. Department of Energy, National Nuclear Security Administration by the University of California, Lawrence Livermore National Laboratory under contract No. W-7405-Eng-48.

\section{References}

[] Bopp, B. W. \& Stencel, R. E. (1981) ApJ, 247, L131
[] Eggleton, P. P. \& Kiseleva, L. (1996) in Evolutionary Processes in Binary Stars eds. Wijers, R. A. M. J., Davies, M. B. \& Tout, C. A., p345

[] Eggleton, P. P. \& KisselevaEggleton, L. (2005) in Binaries in the 21st Century, eds Giménez, A., Guinan, E.F., Niarchos, P. \& Rucinski, S. M. ASS Conf Ser., in press

[] Evans, D. S. \& Fekel, F. C. (1979) ApJ, 228, 497

[] Flannery, B. P. (1976) ApJ, 205, 217

[] Griffin, R. E. M. (2002) AJ, 123, 988

[] Griffin, R. E. M. \& Griffin, R. F. (2000) MN, 319, 1094

[] Griffin, R. F. \& Duquennoy, A. (1993) Obs. 113, 53

[] Hilditch, R. W. (2005) Obs, 125, 72

[] Hoffleit, D. \& Jaschek, C. (1983) 'The Bright Star Catalogue', 4th ed. New Haven: Yale University Observatory)

[] Hummel, C. A., Carquillat, J.-M., Ginestet, N., Griffin, R. F., Boden, A. F., Hajian, A. R., Mozurkewitch, D. \& Nordgren, T. E. (2001) AJ, 121, 1623

[] Hut, P. (1981) A\&A, 99, 126

[] Lucy, L. B. (1976) ApJ, 205, 208

[] Nelson, C. A. \& Eggleton, P. P (2001; NE01) ApJ, 552, 664

[] Pols, O. R., Tout, C. A., Schröder, K.-P., Eggleton, P. P. \& Manners, J. (1997) MN, 289, 869

[] Popper, D. M. (1988) AJ, 96, 1040

[] Pourbaix, D. (1999) A\&A, 348, 127

[] Robertson, J. A. \& Eggleton, P. P. (1977) MN 179, 359

[] Scarfe, C. D., Barlow, D. J., Fekel, 
F. C., Rees, R. F., Lyons, R. W., Bolton, C. T., McAlister, H. A. \& Hartkopf, W. I. (1994) AJ, 107, 1529

[] Schröder, K.-P., Pols, O. R. \& Eggleton, P. P. (1997) MN, 285, 696

[] Terrell, D., Kaiser, D. H., Henden, A. A., Koff, R., West, D., Dvorak, S., Pullen, A. C. \& Stephen, C. P. (2003) AJ, 126, 902

[] Thompson, M. J., ChristensenDalsgaard, J., Miesch, M. S. \& Toomre, J. (2003) ARA\&A, 41, 599.

[] Webbink, R. F. (1976) ApJ, 209, 829

[] Webbink, R. F. (1980) in Close Binary Systems: Observations and Interpretations eds Plavec, M. J., Popper, D. M. \& Ulrich, R. K. IAU Symp. 88, p127

[] Yakut, K. \& Eggleton, P. P. (2005;

YE05) ApJ, 629, 1055 